\title{
Diferenciación de tierras agrícolas en el municipio de Tequisquiapan, Querétaro
}

\section{Differentiation of agricultural lands in the municipality of Tequisquiapan, Querétaro}

\author{
Genaro Aguilar-Sánchez ${ }^{1}$ \\ Universidad Autónoma Chapingo, México
}

\begin{abstract}
Resumen
El municipio de Tequisquiapan, estado de Querétaro, México, ha tenido cambios en el uso de la tierra por el crecimiento urbano. El objetivo es realizar una diferenciación de tierras según el condicionamiento ambiental, para después valorar y clasificar tipos de tierras. La metodología se basa en la clasificación de tierras, propuesta por la (FAO, 1997). Como resultado se obtienen 7 clases se tierras: clase |I, cuya limitante es el clima semiseco, al igual que las clases II y III, donde la profundidad del suelo, son poco limitantes, para la producción agrícola. En las clases IV y V, además, de las piedras, poca profundidad del suelo, clima semiseco, tiene relevancia la inclinación del terreno de más del $15 \%$, que se puede usar para el pastoreo de ganado. En las clases VI y VII, cobra importancia la alta cantidad de rocas , > $50 \%$ de pedregosidad; así como una inclinación mayor del $25 \%$, se recomienda que se usen para la preservación de la vegetación.
\end{abstract}

Palabras clave: Clases de tierras agrícolas, Periodos de crecimiento, Tequisquiapan, México

\begin{abstract}
The municipality of Tequisquiapan, state of Querétaro, Mexico, has suffered changes in land use due to urban growth. The objective of the present research is to differentiate land according to environmental conditioning and then assess and classify land types. The methodology is based on land classification guidelines as proposed by (FAO, 1997). As a result, 7 classes of land are obtained: Class I, whose limitation is semi-dry climate; Classes II and III, where soil depth is non-limiting for agricultural production. Classes IV and V, which in addition to having rocky, shallow depth soils, present a semi-dry climate and over $15 \%$ inclination that make them only suitable for grazing cattle.
\end{abstract}

1 Doctor en Geografía, DCRU- Universidad Autónoma Chapingo. Carretera México-Texcoco Km 38.5. Correo electrónico: g_aguila@correo.chapingo.mx https://orcid.org/0000-0003-1518-0801 
In classes VI and VII, the substantial stoniness ( $>50 \%$ ) character of soils as well as greater than $25 \%$ slopes result in their recommendation as vegetation preservation land.

Keywords: Agricultural land classes; Growth periods; Tequisquiapan; Mexico.

\section{Introducción}

El uso de la tierra a través del tiempo ha cambiado debido a nuevas actividades, como el cambio fines agrícolas por actividades industriales y recreativas, es decir, actividades turísticas. El estado de Querétaro, por la cercanía al centro metropolitano más grande del país, formado por la Ciudad de México, antes Distrito Federal, y el Estado de México, y otros estados como Hidalgo, Morelos, Tlaxcala, Guanajuato y Michoacán, ha tenido grandes cambios en el uso de la tierra de sus municipios, en particular en los municipios más cercanos a los centros conurbados, como es el caso del Municipio de Tequisquiapan, por lo que surge la problemática de indagar la situación actual del uso de sus tierras, para verificar si el uso de los recursos naturales como suelo, agua, relieve, clima, que le dan contenido a cada clase de tierra tiene o no el uso adecuado.

Además, el cambio de uso de la tierra en los últimos 30 años, por las políticas neoliberales ha traído como consecuencia cambios en la tenencia de la tierra, traslado paulatino de la administración y manejo del agua de las instituciones de gobierno a los grupos de productores, además, del decremento del área agrícola, que se manifiesta en la disminución de la producción, y la mayor importación de productos básicos como el maíz y el frijol, lo anterior obliga a valorar la situación del uso de la tierra, así como de la producción a nivel municipal y de sus comunidades

En este trabajo se usa el concepto de tierra, que es más amplio que el de suelo, ya que el concepto de tierra engloba al relieve, geología, suelo, vegetación, con relativa estabilidad y homogeneidad, con un clima definido, es decir, se incluye la parte terrestre de una unidad cartográfica, así como la parte de la atmosfera, como es el clima, con su temperatura, periodo de lluvias, heladas, sequias. Así como las actividades productivas pasadas y presentes que ha realizado el hombre. (Ortiz y Cuanalo, 1978).

El objetivo general es realizar una delimitación de unidades cartográficas, agroambientes, relativamente homogéneas, según el condicionamiento ambiental, para después valorar y diferenciar clases de tierras agrícolas. 


\section{Marco Teórico}

El suelo es uno de los componentes de la tierra, en el que desarrollan las actividades económicas de la sociedad, sin embargo, existen situaciones en las que este recurso se utiliza de manera inadecuada provocando procesos de degradación, lo cual implica una disminución de su capacidad para producir bienes o prestar servicios para sus beneficiarios y perdida de la salud edáfica (FAO, 1997). Uno de los procesos de degradación de suelo es la erosión hídrica en la cual influyen de manera directa varios factores, tales como el nivel de precipitación, la permeabilidad definida por la clase textural de los suelos presentes, la pendiente, la cubierta vegetal y en el caso de suelos de uso agrícola-pecuarios las obras de conservación. Por ello es muy importante incorporar la estimación de erosión en la evaluación agroecológica de tierras, ya que nos permiten conocer el grado de influencia que tienen los diferentes factores y de esta manera proponer estrategias para atenuarlas o potenciarlas según sea el caso.

También es importante estimar la humedad que existe en el suelo, producto de la lluvia de temporal, así como la existencia de heladas, para obtener el periodo de crecimiento, PC., es decir, la duración en meses y días, que tienen los cultivos para completar su ciclo agrícola, desde la germinación de la semilla hasta la maduración del producto. Como lo indican (Pájaro y Ortiz 1988) y (Aguilar Sánchez, 1995)

La clasificación de tierras agrícolas es una propuesta realizada por la (FAO, 1997), a través del trabajo Zonificación agroecológica de tierras de la FAO, donde propone diferenciar las tierras en 8 clases. Aunque también en México existe el trabajo del Instituto Nacional de Estadística y Geografía e Informática, (INEGI, 2005), con su propuesta de uso potencial de suelos, donde indica que las tierras se deben diferenciar de acuerdo al uso potencial más adecuado. Propone clases de capacidad de uso agrícola con 5 clases, para uso pecuario 5 clases y para uso forestal 4 clases. La clasificación que se usa en este trabajo es la de FAO, ya que el uso actual de la tierra está muy mezclada en los diversos territorios de México, y el estado de Querétaro no es la excepción

\section{Metodología}

Para la delimitación de Ambientes agrícolas se usó la Metodología del condicionamiento ambiental en la agricultura, propuesta por, Romero P.J, et al. (2003), a escala 1: 500.000 
Para diferenciar la clase de tierras se usó la propuesta de Zonificación agroecológica de tierras de la FAO (1997), la que indica la diferenciación de ocho clases de tierra. Encada ambiente agrícola diferenciado, se obtiene el periodo de crecimiento en base a la propuesta de Pájaro y Ortiz (1988)

\section{Procedimiento de Estimación del Periodo de Crecimiento}

Cuando se cuenta con la información básica para el establecimiento del PC de crecimiento se realizan los siguientes pasos:

a) Se graficaron los valores mensuales de precipitación, evapotranspiración potencial (estimada a partir de $0.8 \mathrm{Ev}$ ), la mitad de la evapotranspiración potencial, ETP, y el .33 \% de la ETP corresponde al Punto de Marchitamiento Permanentes (PMP), estableciéndose una curva para cada dato (P, ETP, 0.5 ETP y PMP).

b) Se estableció el inicio y terminación de los periodos de crecimiento de acuerdo a las normas indicadas en los conceptos básicos y se definió el tipo de periodo de crecimiento (normal, intermedio, seco todo el año, húmedo todo el año).

c) Se determinó la temperatura media o mínima del periodo menor a $6.5^{\circ}$ C, (Matías, L. F. O. \&. G. F.Centro Nacional dePrevención de Desastres., 2001) y

d) Se estableció la duración del periodo de crecimiento, restando el número de días con temperaturas menores a $6.5^{\circ} \mathrm{C}$ al número de días determinados con disponibilidad de humedad.

En caso que no exista un periodo húmedo y humedad almacenada, el final del periodo de crecimiento coincide con la terminación de la estación lluviosa.

\section{Periodo de Crecimiento}

Inicio del periodo de crecimiento: Coincide con el comienzo de la estación lluviosa. Específicamente cuando $\mathrm{P}=0.5$ ETP y después $\mathrm{P}>0.5$ ETP.

Periodo Húmedo: Un periodo húmedo es el intervalo de tiempo, en el cual la precipitación es mayor a la evapotranspiración potencial ( $\mathrm{P}>\mathrm{ETP}$ ).

Inicio de periodo húmedo cuando $\mathrm{P}=\mathrm{EPT}$ (antes $\mathrm{P}<\mathrm{ETP}$ y después $\mathrm{P}>\mathrm{ETP}$ ). Final del periodo húmedo cuando $\mathrm{P}=\mathrm{EPT}$ (antes $\mathrm{P}>\mathrm{ETP}$ 
y después $\mathrm{P}<\mathrm{ETP}$ ). Final de la estación lluviosa: Termina cuando $\mathrm{P}<$ 0.5 ETP).

Terminación del periodo de crecimiento: Cuando existe periodo húmedo y Humedad almacenada, al final de la estación lluviosa se considera el número de días hasta que se agotan las reservas de humedad del suelo (no pueden ser mayores a $100 \mathrm{~mm}$ ).

Cuando no existe periodo húmedo y humedad almacenada, el final del periodo de crecimiento coincide con la terminación de la estación lluviosa.

\section{Modelos de Regresión}

Se utilizaron modelos de regresión que permites estimar estos periodos de crecimiento en cualquier región con sólo disponer del dato de la precipitación total anual (PT), como este:

$\mathrm{PC}=0.24089$ PT $-0.0000372 \mathrm{PT}^{2}-33.1019$; donde: $\mathrm{PC}=$ Periodo de crecimiento, en días $\mathrm{PT}=$ Precipitación total anual, en $\mathrm{mm}$

Con la finalidad de determinar el inicio del periodo de crecimiento (IPC) en días julianos, se desarrolló el siguiente modelo:

IPC $=210.3526-0.3976$ PC; donde:

IPC $=$ inicio del periodo de crecimiento en días julianos ( 1 para el 1 de enero y 365 para el 31 de diciembre). $\mathrm{PC}=$ Periodo de crecimiento en días estimado por el modelo anterior

\section{Periodo de Heladas}

Se utilizó un modelo para determinar la duración del periodo libre de heladas (PLH), la fecha de la última helada (UH) y la fecha de la primera helada $(\mathrm{PH})$ :

$\mathrm{PLH}=1.7713+31.0214 \mathrm{Tmin}-0.6361(\mathrm{Tmin})^{2}$; donde: $\mathrm{PLH}=\mathrm{Pe}-$ riodo libre de heladas, en días

Tmin $=$ Temperatura mínima media anual, en ${ }^{\circ} \mathrm{C}$

$\mathrm{UH}=225.3605-0.7396 \mathrm{PLH}+0.0004385(\mathrm{PLH})^{2}$; donde: $\mathrm{UH}=$ fecha de la última helada en días julianos

$\mathrm{PLH}=$ período libre de heladas, en días

$\mathrm{PH}=229.5781+0.2262 \mathrm{PLH}+0.0005098(\mathrm{PLH})^{2}$; donde: $\mathrm{PH}=$ fecha de la primera helada en días julianos

$\mathrm{PLH}=$ Periodo libre de heladas, en días. 


\section{Información utilizada}

El periodo de crecimiento se hizo a partir de la información climática que proporcionan el Sistema Meteorológico Nacional y CONAGUA, ubicando seis estaciones climatológicas dentro de los límites y la periferia del municipio de Tequisquiapan que corresponden a las estaciones; 22031 Presa Paso de Tablas, periodo 1951-2010; Estación 22025 Presa Centenario, periodo 1951-2010; Estación 22042 La llave, periodo 1951-2010; estación 22009 La palma periodo 1951-2010; estación 22034 Villa Bernal 1951-2010; estación 22046 Nogales periodo 1951-2010. Posteriormente, se extrajeron los datos promedios mensuales y anuales de temperatura y precipitación.

Para indagar el uso actual se recurrirá al trabajo en campo para aplicar entrevistas semiabiertas con los productores de las diversas clases de tierras, como indican (Rojas, 2012, Lafuente \&Marìn, 2008).

\section{Calculo de la precipitación mediante el método de Thiessen}

El cálculo de la precipitación dentro del municipio, fue mediante el método de los polígonos de Thiessen, basándose en las precipitaciones en cada estación en función de un área de influencia mediante el Programa ArcGIS 9.31. Para la determinación de las zonas de influencia de las estaciones, se unieron las estaciones trazando las mediatrices de los triángulos y uniéndolas convenientemente, formando polígonos limitados exteriormente por los límites de municipio, cuyas superficies corresponden a las zonas de influencia de cada estación ((Sánchez, F., 2016, 2016 Noviembre) y (Becerra Moreno, 1999)):

$$
\begin{aligned}
& \mathrm{P}=1 / \mathrm{A} \Sigma^{\mathrm{n}} A i P i \\
& i=1
\end{aligned}
$$

Donde $\mathrm{A} i=$ Área del polígono en la estación $i$

$\mathrm{P} i=$ Precipitación en estación $i$

En la estimación de la erosión hídrica se usa la Ecuación Universal de Pérdida de Suelo, EUPS, propuesta por (Wischmeir, 1978), modificada y mejorada por (Colegio de Postgraduados, 1991). Dicha metodología combina la erosividad de la lluvia, la erodabilidad del suelo, la longitud y pendiente del terreno, las prácticas de manejo del terreno y, la presencia o ausencia de obras de conservación, (Fernández Reynoso, 2015) 


\section{Resultados}

El estado de Querétaro es uno de los estados más pequeños del país, sin embargo, también es uno de los más heterogéneos. La cantidad de atractivos que encierra lo convierten en un estado fascinante, dinámico, colorido y diverso. Su capital es Santiago de Querétaro, tiene 18 municipios en una extensión de $11.699 \mathrm{~km}^{2}$, o sea un $0.60 \%$ del territorio nacional, tiene una población de 2’038.372 habitantes, el $1.7 \%$ del total del país. Su distribución es $70 \%$ urbana y $30 \%$ rural; a nivel nacional el dato es de 78 y $22 \%$ respectivamente. El sector que más aporta al PIB es el sector terciario, con un $2.2 \%$ anual. Respecto a los hablantes de lenguas indígenas, 2 de cada 100 personas. A nivel nacional 7 de cada 100 personas hablan lengua indígena. (INEGI, 2015)

La regionalización agropecuaria del Estado de Querétaro se deriva de la Ley de Distritos de Desarrollo Rural (1988), (SEDEA-Qro., 2018), que determina formar los Distritos de Desarrollo Rural como zonas con características ecológicas y socio-económicas homogéneas para la actividad agropecuaria, forestal, de las agroindustrias y de acuacultura bajo condiciones de riego, de drenaje y de temporal con objeto de planear, fomentar y promover el Desarrollo Rural Integral. Para lo anterior se consideró la división política municipal, la sub-regionalización establecida por el Comité Estatal para la Planeación del Desarrollo, las condiciones agroecológicas y agroeconómicas municipales, el uso del suelo, la infraestructura de irrigación y drenaje, así como las vías de comunicación e infraestructura de apoyo. Estas regiones son consideradas unidades de desarrollo económico y social circunscritas a un espacio territorial, para articular y dar coherencia a las políticas de desarrollo rural, se clasifica en cuatro regiones agropecuarias, la Región Jalpan comprendida por los municipios Pinal de Amoles, Arroyo Seco, Jalpan de Sera y Landa de Matamoros, Región Cadereyta con los municipios Cadereyta de Montes, Colón, Ezequiel de Montes, Peñamiller, San Joaquín y Tolimán, Región Querétaro que contiene los municipios Corregidora, Huimilpan, El Marqués y Querétaro. Así como nuestra región de interés, San Juan del Río que comprende los municipios de Amealco de Bonfil, Pedro Escobedo, San Juan del Río y Tequisquiapan, es la más productiva y en general cuenta con mejor infraestructura de apoyo: carreteras, presas, maquinaria, organizaciones de productores y centros de consumo y comercialización. Características generales de los recursos naturales en Tequisquiapan 
De acuerdo al INEGI (1995). El municipio de Tequisquiapan está sujeto mayormente a climas áridos templado, semiárido templado con estación de lluvias bien marcada y altas temperaturas durante verano. La lluvia invernal no rebasa el 5 por ciento del total de la precipitación anual. En la parte baja de la zona delimitada se ubica un clima templado con lluvias invernales superiores al 10 por ciento. En el área predominan temperaturas medias anuales entre 16 y $18^{\circ} \mathrm{C}$. En estos climas la precipitación media anual es relativamente escasa entre los 400 - $600 \mathrm{~mm}$ anuales.

El territorio del municipio de Tequisquiapan (INAFED, 2010) se encuentra situado en la altiplanicie mexicana, en las estribaciones australes de la Sierra Gorda, se localizan zonas relativamente planas que forman pequeños valles y planicies que se han convertido en áreas de cultivo. Por el sur del territorio entra la corriente superficial del río San Juan, nombre que toma a su paso por la localidad de San Juan del Río; este caudal alimenta la Presa Centenario, sigue su curso al noreste a la Presa Paso de Tablas, límite con el Estado de Hidalgo (CONAGUA, 2016). En la mayor parte de la región afloran rocas ígneas extrusivas, y sedimentarias continentales recientes. La vegetación es de matorral espinoso, nopaleras y relictos de selva baja caducifolia. Con la conjugación de relieve, geología, clima y vegetación, en las tierras de Tequisquiapan se encuentra suelos que se clasifican como regosol, R., feozem haplico, Hh y luvico, Hl, vertisol pelico, Vp., y rendzina, E.

El municipio Tequisquiapan, palabra de origen náhuatl que significa "lugar de tequesquite", ya que está formado por las palabras tequexquitl: tequesquite; apan: lugar. Tequisquiapan en término mexicano quiere decir tierra baja tequexquitosa, donde abunda el salitre que se hace visible a la vista (Edo de Qurètaro, 2016), y viendo así tierra baja de suelo de Tequisquiapan y abundan muchos parajes de su centro, fuera de él y en sus riveras con esta materia, de ahí viene el nombre de Tequisquiapan". Se localiza en la parte Sureste del Estado de Querétaro Cuenta con una superficie de 343.6 kilómetros cuadrados y representa el $2.3 \%$ de la superficie del Estado. Tequisquiapan se encuentra situado en la altiplanicie mexicana, en las estribaciones australes de la Sierra Gorda, y donde se localizan zonas relativamente planas que forman pequeños valles y planicies que se han convertido en áreas de cultivo. Mapa 1. 


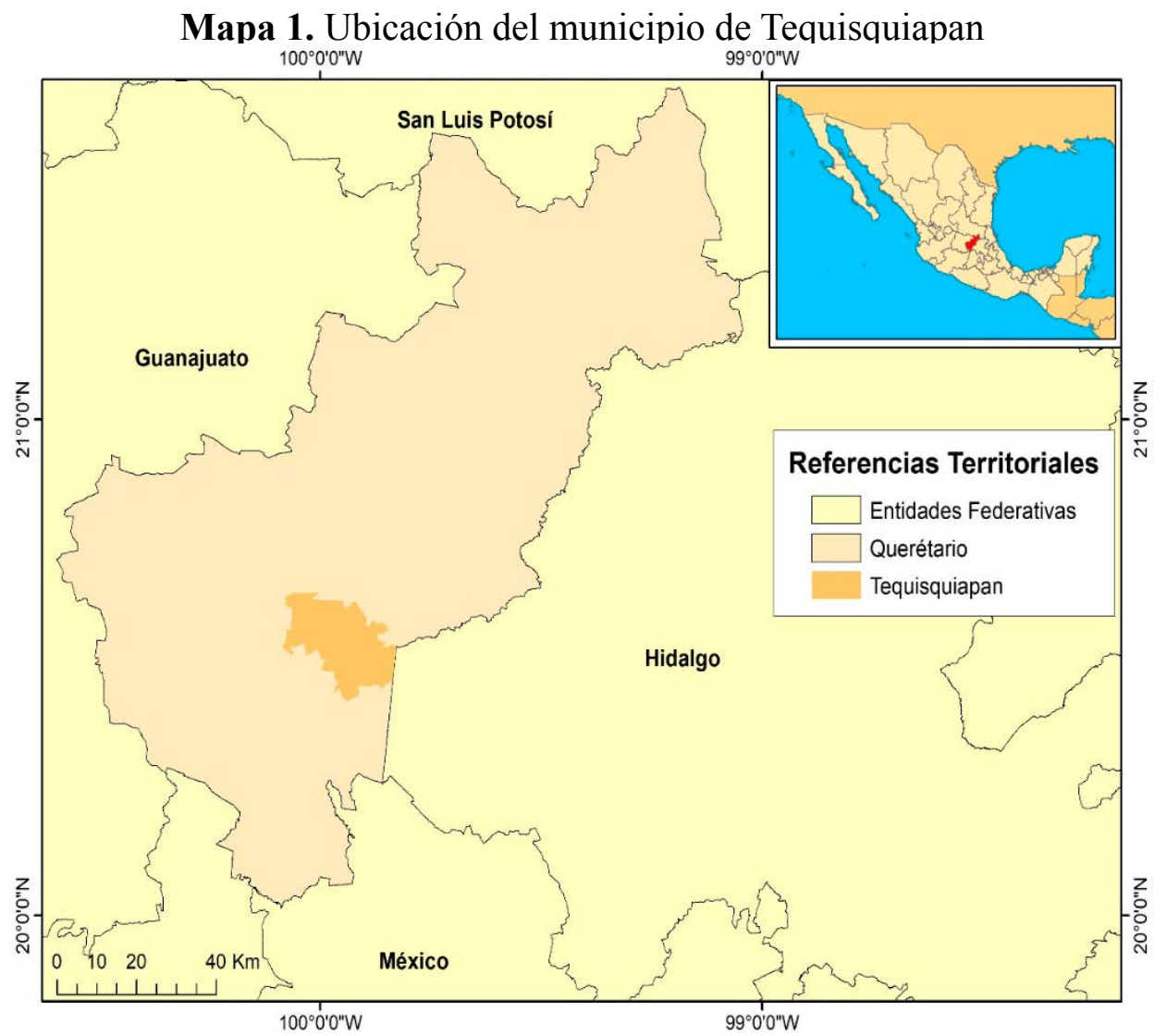

Por el Sur del territorio entra la corriente superficial del río San Juan, nombre que toma a su paso por la localidad de San Juan del Río; este caudal alimenta la Presa Centenario, sigue su curso al Noreste a la Presa Paso de Tablas, límite con el Estado de Hidalgo. En este sitio se aprovechaba el caudal hidráulico para producir energía eléctrica, lugar donde se encuentra instalada una planta hidroeléctrica fuera de uso. Río abajo se une al río Moctezuma y desemboca en el Golfo de México. Presenta tanto agricultura de riego como de temporal, tiene una vegetación de matorral, principalmente, seguido de bosque de encino, pastizal inducido y mezquital, (Universidad Autonòma de Querètaro, 1995).

El clima de Tequisquiapan es templado, con dos épocas bien definidas al año. La precipitación pluvial se da en los meses de junio a octubre, en tanto que la temporada de secas comprende los meses de noviembre a 
abril, en mayo lluvias aisladas. El índice promedio de precipitación pluvial es de 78 días con una media anual de $511.8 \mathrm{~mm}$. Los veranos son cálidos y el invierno no muy extremo, ya que la temperatura media anual es de $17.5^{\circ} \mathrm{C}$. Los vientos dominantes son en dirección Noreste -Suroeste. Las heladas se presentan en los meses de octubre a febrero y esporádicamente en marzo y fines de septiembre. El índice promedio es de 18 días al año. En el año 2003, el promedio anual se rebasó en forma considerable hasta alcanzar los $600 \mathrm{~mm}$. La fórmula climática es BS1 kw(w) representa un 99 $\%$ de la superficie del municipio con $367.99 \mathrm{~km}^{2}$ y BS1 hw apenas un $1 \%$ del municipio, con $1.62 \mathrm{~km}^{2}$.

\section{Periodos de crecimiento}

Para el cálculo del periodo de crecimiento (PC), se obtuvieron datos de las estaciones meteorológicas de CONAGUA cercanas a Tequisquiapan, los cálculos se basaron en la metodología de estimación del periodo de crecimiento por disponibilidad de agua y libre de heladas para la República Mexicana, Pájaro y Ortiz (1988). Los resultados se concentran en el cuadro 3.

\section{Agroambientes del municipio de tequisquiapan}

Dentro del municipio se delimitaron 17 agroambientes, considerando la fisiografía, geología, clima, tipo de suelo, vegetación y uso actual dentro de la región, obteniéndose un mapa con los polígonos de cada unidad de estudio (Mapa 2). 
Mapa 2. Mapa de Agroambientes y sitios de muestreo, municipio de

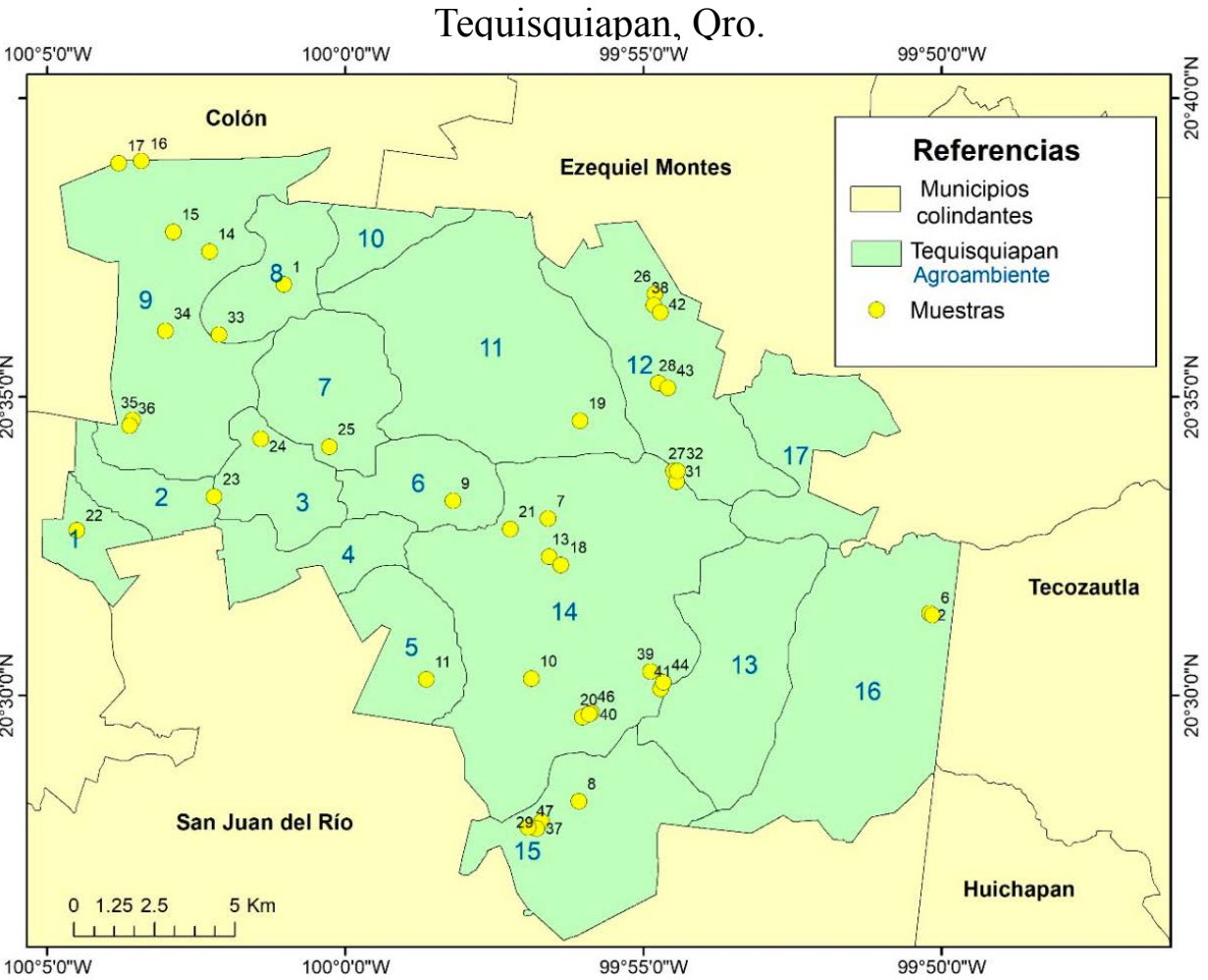

También en el mapa 2, se observan los sitios de muestreo por cada agroambiente. En base a la información obtenida en gabinete y con el trabajo en campo se elaboraron cuadros, donde se concentra la información, que se muestra a continuación

En el cuadro 1, se observa un total de 17 agroambientes, los grandes son: 14,9 y 11 , los más pequeños tienen los números 1,10 y 2 . La geología que domina es de rocas ígneas extrusivas, como el basalto. En las unidades de suelo domina el Feozem haplico, Hh, y Feozem luvico, Hl; con pocos agroambientes con suelo Vertisol pelico, Vp; de manera secundaria están los Litosol, I, y Regosol, R. El clima dominante es semiseco. La vegetación es de Matorral craucicable, pastizal y, poco Bosque de encino. El uso agrícola actual, en el ciclo de primavera-verano se compone de siembra de maíz, pocas hortalizas en invernaderos. Y en el ciclo de otoño-invierno se siembra cebada y avena. 
Genaro Aguilar-Sánchez

Diferenciación de tierras agrícolas en el municipio de Tequisquiapan, Querétar

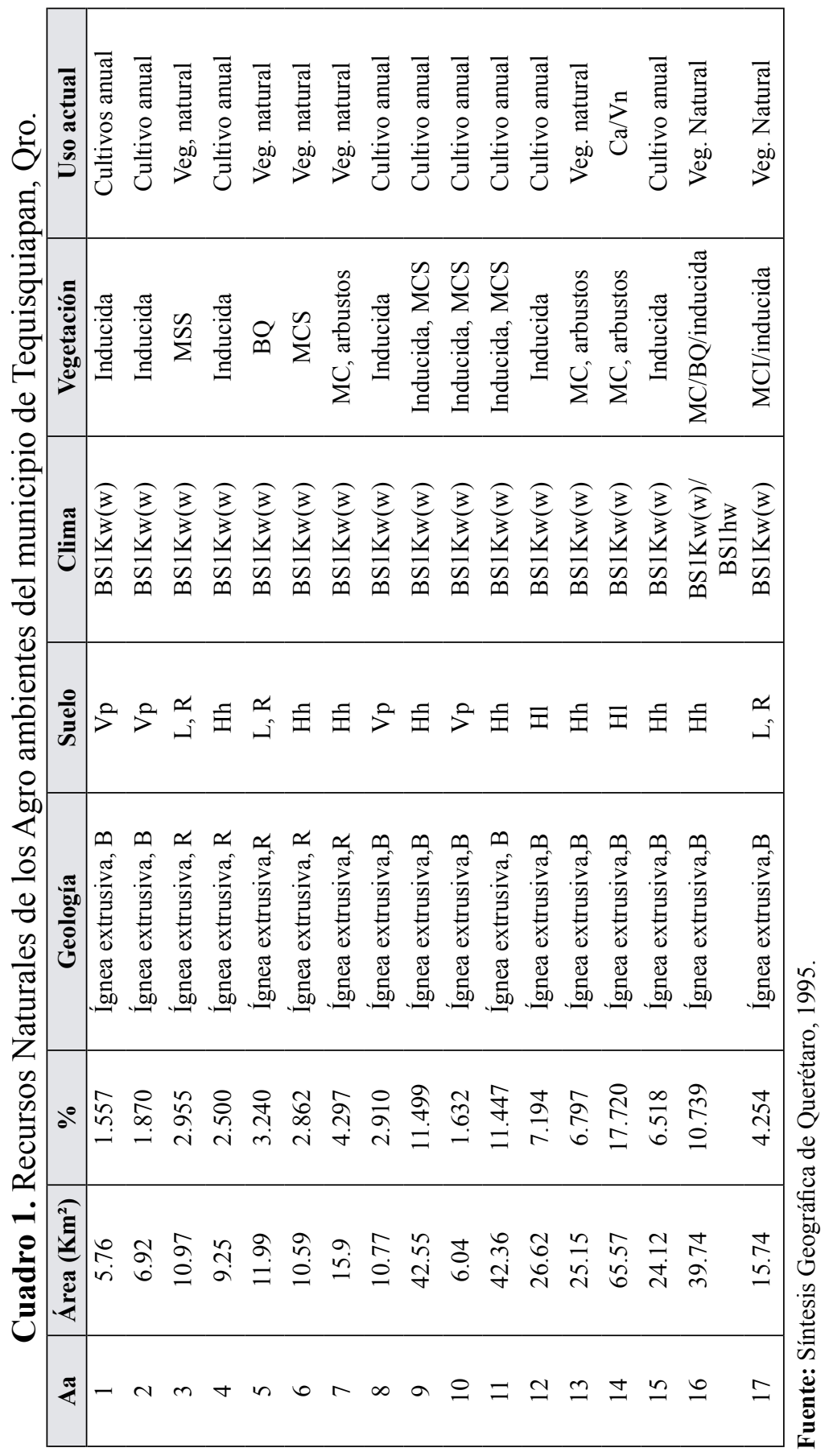

132 Revista Geográfica de América Central. No 65(2)

ISSN 1011-484X • e-ISSN 2215-2563 / Julio-diciembre 2020

Doi: http://dx.doi.org/10.15359/rgac.65-2.5 
Aa: Agroambiente, B: basalto, R: riolita; $\mathrm{BS} 1 \mathrm{Kw}(\mathrm{w})$ : semiseco, MC: matorral crausicaule.

BQ: bosque de encino, MSS: matorral subtropical subinerme, MCI: matorral craucicable inerme,

MCS: matorral crausicaule subinerme. Ca: cultivo anual, Vn: vegetación natural

Además, se elaboró el cuadro 2, donde se concentró la información de las características edáficas de cada agroambiente y se agruparon en base a su similitud.

Cuadro 2. Características edáficas de los agroambientes del municipio de Tequisquiapan

\begin{tabular}{|c|c|c|c|c|c|c|c|c|}
\hline Aa & Textura & Prof. & $\begin{array}{c}\text { Fase } \\
\text { física }\end{array}$ & M.O & $\mathbf{p}^{\mathbf{H}}$ & Piedras & Estructura & $\begin{array}{c}\mathbf{m} \\
\mathbf{\%}\end{array}$ \\
\hline 1 & Arcillosa & $>50$ & $\mathrm{Dp}$ & mucha & $7-8$ & pocas & Bp & $1-5$ \\
2 & Arcillosa & $>50$ & $\mathrm{Dp}$ & mucha & $7-8$ & pocas & Bp & $1-5$ \\
3 & Arenosa & $<20$ & $\mathrm{~Np}$ & poca & 8 & muchas & granular & $5-10$ \\
4 & $\mathrm{Ma}$ & $21-50$ & $\mathrm{D}$ & media & 7 & media & Bs & $1-5$ \\
5 & Arenoso & $21-30$ & $\mathrm{~Np}$ & poca & 8 & muchas & granular & $>11$ \\
6 & $\mathrm{Ma}$ & $21-50$ & $\mathrm{D}$ & media & $6-7$ & muchas & Bs & $>11$ \\
7 & $\mathrm{Ma}$ & $21-50$ & $\mathrm{D}$ & mucha & 7 & media & Bs & $>11$ \\
8 & Arcillosa & $>50$ & $\mathrm{Dp}$ & mucha & $7-8$ & pocas & Bp & $1-5$ \\
9 & $\mathrm{Ma}$ & $21-50$ & $\mathrm{D}$ & media & 7 & media & Bs & $1-5$ \\
10 & Arcillosa & $>50$ & $\mathrm{Dp}$ & mucha & $7-8$ & pocas & Bp & $1-5$ \\
11 & $\mathrm{Ma}$ & $21-50$ & $\mathrm{D}$ & media & $6-7$ & media & Bs & $1-5$ \\
12 & $\mathrm{Ma}$ & $>50$ & $\mathrm{Dp}$ & mucha & $6-7$ & media & Bs & $1-5$ \\
13 & $\mathrm{Ma}$ & $21-50$ & $\mathrm{D}$ & mucha & $6-7$ & media & Bs & $>11$ \\
14 & $\mathrm{Ma}$ & $>50$ & $\mathrm{Dp}$ & media & $6-7$ & media & Bs & $1-5$ \\
15 & $\mathrm{Ma}$ & $21-50$ & $\mathrm{D}$ & mucha & $6-7$ & media & Bs & $>11$ \\
16 & $\mathrm{Ma}$ & $21-50$ & $\mathrm{D}$ & mucha & 7 & media & Bs & $>11$ \\
17 & Arenoso & $<20$ & $\mathrm{~Np}$ & poca & 8 & muchas & granular & $>11$ \\
\hline
\end{tabular}

Fuente: trabajo en campo

Aa: agroambiente, m: pendiente, Ma: migajón arcilloso, D: Dúrica, Dp: Dúrica profunda,

Bp: bloque prismático, Bloque subangular 
La ubicación de los sitios de muestreo, por cada agroambiente y las características de los periodos de crecimiento, PC., se detallan en el cuadro 3. Donde se muestra que el PC., que predomina es del 28 de mayo al 29 de septiembre, es decir, se tienen 124 días con humedad suficiente y sin problemas de heladas. Para sembrar cultivos de ciclo medio como maíz, frijol, avena, haba, etc. En segundo lugar, están los agroambientes donde dura el ciclo del PC, del 2 de junio al 13 de octubre, con 133 días con humedad y sin heladas. Donde se siembra cultivo de maíz de ciclo intermedio. En tercer lugar, está el PC., del 16 de mayo al 14 de octubre, 150 días, para sembrar variedades de maíz u otros cultivos, de ciclo largo. Aunque en estos sitios del agroambiente 9, ubicado a más de $2000 \mathrm{msnm}$, se corre el riesgo de una helada a inicios de octubre, pero los cultivos de 140-150 días tienen muchas posibilidades de prosperar. En el anterior, faltan los sitios 3,4 y 5 , porque son sitios que quedan fuera del municipio de estudio.

Cuadro 3. Ubicación geográfica de los sitios de muestreo y agroambientes

\begin{tabular}{|c|c|c|c|c|c|}
\hline \multirow{2}{*}{ Punto } & \multicolumn{2}{|c|}{ Coordenadas } & \multirow{2}{*}{ Aa } & \multirow{2}{*}{$\begin{array}{l}\text { Altitud } \\
(\mathrm{msnm})\end{array}$} & \multirow{2}{*}{$\begin{array}{c}\text { Periodo } \\
\text { De Crecimiento }\end{array}$} \\
\hline & Latitud (Norte) & Longitud (Oeste) & & & \\
\hline 1 & $20^{\circ} 36^{\prime} 53.1^{\prime \prime}$ & $100^{\circ} 1^{\prime} 1.8^{\prime \prime}$ & 8 & 2093 & $25-5$ al $29-9$ \\
\hline 2 & $20^{\circ} 31^{\prime} 22.8^{\prime \prime}$ & $99^{\circ} 50^{\prime} 12.3^{\prime \prime}$ & 16 & 1956 & $28-5$ al $29-9$ \\
\hline 6 & $20^{\circ} 31^{\prime} 20.9^{\prime \prime}$ & $99^{\circ} 50^{\prime} 09.1^{\prime \prime}$ & 16 & 1941 & $28-5$ al 29-9 \\
\hline 7 & $20^{\circ} 32^{\prime} 57.8^{\prime \prime}$ & $99^{\circ} 56^{\prime} 36.1^{\prime \prime}$ & 14 & 1920 & $28-5$ al 29-9 \\
\hline 8 & $20^{\circ} 28^{\prime} 13.6^{\prime \prime}$ & $99^{\circ} 56^{\prime} 05^{\prime \prime}$ & 15 & 1916 & $28-5$ al 29-9 \\
\hline 9 & $20^{\circ} 33^{\prime} 15.5^{\prime \prime}$ & $99^{\circ} 58^{\prime} 11.8^{\prime \prime}$ & 6 & 1925 & $28-5$ al 29-9 \\
\hline 10 & $20^{\circ} 30^{\prime} 17^{\prime \prime}$ & $99^{\circ} 56^{\prime} 52.7^{\prime \prime}$ & 14 & 1917 & $28-5$ al 29-9 \\
\hline 11 & $20^{\circ} 30^{\prime} 16.1^{\prime \prime}$ & $99^{\circ} 58^{\prime} 38.5^{\prime \prime}$ & 5 & 2222 & $07-6$ al $12-9$ \\
\hline 13 & $20^{\circ} 32^{\prime} 19.6^{\prime \prime}$ & $99^{\circ} 56^{\prime} 35.1^{\prime \prime}$ & 14 & 1919 & $28-5$ al 29-9 \\
\hline 14 & $20^{\circ} 37^{\prime} 26^{\prime \prime}$ & $100^{\circ} 02^{\prime} 16.7^{\prime \prime}$ & 9 & 2051 & $16-5$ al $14-10$ \\
\hline 15 & $20^{\circ} 37^{\prime} 45.7^{\prime \prime}$ & $100^{\circ} 02^{\prime} 53.0^{\prime \prime}$ & 9 & 2040 & $16-5$ al $14-10$ \\
\hline 16 & $20^{\circ} 38^{\prime} 57.2^{\prime \prime}$ & $100^{\circ} 03^{\prime} 25.2^{\prime \prime}$ & 9 & 2026 & $16-5$ al $14-10$ \\
\hline 17 & $20^{\circ} 38^{\prime} 54.8^{\prime \prime}$ & $100^{\circ} 03^{\prime} 47.9^{\prime \prime}$ & 9 & 2043 & $16-5$ al $14-10$ \\
\hline 18 & $20^{\circ} 32^{\prime} 11^{\prime \prime}$ & $99^{\circ} 56^{\prime} 23^{\prime \prime}$ & 14 & 1907 & $28-5$ al 29-9 \\
\hline 19 & $20^{\circ} 34^{\prime} 36^{\prime \prime}$ & $99^{\circ} 56^{\prime} 4^{\prime \prime}$ & 11 & 1742 & $02-6$ al $13-10$ \\
\hline 20 & $20^{\circ} 29^{\prime} 38.5^{\prime \prime}$ & $99^{\circ} 56^{\prime} 01.68^{\prime \prime}$ & 14 & 1900 & $28-5$ al 29-9 \\
\hline 21 & $20^{\circ} 32.7^{\prime} 05.1^{\prime \prime}$ & $99^{\circ} 57^{\prime} 14.1^{\prime \prime}$ & 14 & 1910 & $28-5$ al 29-9 \\
\hline 22 & $20^{\circ} 32^{\prime} 46^{\prime \prime}$ & $100^{\circ} 4^{\prime} 30^{\prime \prime}$ & 1 & 1903 & $15-5$ al $13-10$ \\
\hline 23 & $20^{\circ} 33^{\prime} 20^{\prime \prime}$ & $100^{\circ} 2^{\prime} 12^{\prime \prime}$ & 3 & 1911 & $28-5$ al 29-9 \\
\hline 24 & $20^{\circ} 34^{\prime} 17.7^{\prime \prime}$ & $100^{\circ} 01^{\prime} 25.2^{\prime \prime}$ & 3 & 1934 & $28-5$ al 29-9 \\
\hline
\end{tabular}


Genaro Aguilar-Sánchez

Differentiation of agricultural lands in the municipality of Tequisquiapan, Querétaro

\begin{tabular}{|c|c|c|c|c|c|}
\hline \multirow{2}{*}{ Punto } & \multicolumn{2}{|c|}{ Coordenadas } & \multirow{2}{*}{ Aa } & \multirow{2}{*}{$\begin{array}{l}\text { Altitud } \\
\text { (msnm) }\end{array}$} & \multirow{2}{*}{$\begin{array}{c}\text { Periodo } \\
\text { De Crecimiento }\end{array}$} \\
\hline & Latitud (Norte) & Longitud (Oeste) & & & \\
\hline 25 & $20^{\circ} 34^{\prime} 10^{\prime \prime}$ & $100^{\circ} 0^{\prime} 16^{\prime \prime}$ & 7 & 1983 & $02-6$ al $13-10$ \\
\hline 26 & $20^{\circ} 36^{\prime} 43.4^{\prime \prime}$ & $99^{\circ} 54^{\prime} 48.5^{\prime \prime}$ & 12 & 1958 & $02-6$ al $13-10$ \\
\hline 27 & $20^{\circ} 33^{\prime} 45.7^{\prime \prime}$ & $99^{\circ} 54^{\prime} 29.9^{\prime \prime}$ & 12 & 1893 & $02-6$ al $13-10$ \\
\hline 28 & $20^{\circ} 35^{\prime} 14.1^{\prime \prime}$ & $99^{\circ} 54^{\prime} 45.1^{\prime \prime}$ & 12 & 1910 & $02-6$ al $13-10$ \\
\hline 29 & $20^{\circ} 27^{\prime} 53.3^{\prime \prime}$ & $99^{\circ} 56^{\prime} 42.4^{\prime \prime}$ & 15 & 1966 & $28-5$ al $29-9$ \\
\hline 31 & $20^{\circ} 33^{\prime} 35.1^{\prime \prime}$ & $99^{\circ} 54^{\prime} 26.9^{\prime \prime}$ & 14 & 1893 & $28-5$ al 29-9 \\
\hline 32 & $20^{\circ} 33^{\prime} 45.5^{\prime \prime}$ & $99^{\circ} 54^{\prime} 25.8^{\prime \prime}$ & 12 & 1888 & $02-6$ al $13-10$ \\
\hline 33 & $20^{\circ} 36^{\prime} 02.7^{\prime \prime}$ & $100^{\circ} 02^{\prime} 07.3^{\prime \prime}$ & 8 & 1976 & $02-6$ al $13-10$ \\
\hline 34 & $20^{\circ} 36^{\prime} 06.18^{\prime \prime}$ & $100^{\circ} 3^{\prime} 1^{\prime \prime}$ & 9 & 1978 & $02-6$ al $13-10$ \\
\hline 35 & $20^{\circ} 34^{\prime} 37^{\prime \prime}$ & $100^{\circ} 03^{\prime} 34^{\prime \prime}$ & 9 & 1952 & $02-6$ al $13-10$ \\
\hline 36 & $20^{\circ} 34^{\prime} 31^{\prime \prime}$ & $100^{\circ} 3^{\prime} 37^{\prime \prime}$ & 9 & 1942 & $02-6$ al $13-10$ \\
\hline 37 & $20^{\circ} 27^{\prime} 46.3^{\prime \prime}$ & $99^{\circ} 56^{\prime} 47.3^{\prime \prime}$ & 15 & 1920 & $28-5$ al $29-9$ \\
\hline 38 & $20^{\circ} 36^{\prime} 32^{\prime \prime}$ & $99^{\circ} 54^{\prime} 49.5^{\prime \prime}$ & 12 & 1928 & $02-6$ al $13-10$ \\
\hline 39 & $20^{\circ} 30^{\prime} 24.1 "$ & $99^{\circ} 54^{\prime} 53.1^{\prime \prime}$ & 14 & 1916 & $29-5$ al $29-9$ \\
\hline 40 & $20^{\circ} 29^{\prime} 42.8^{\prime \prime}$ & $99^{\circ} 55^{\prime} 52.4^{\prime \prime}$ & 14 & 1887 & $29-5$ al $29-9$ \\
\hline 41 & $20^{\circ} 30^{\prime} 6.8^{\prime \prime}$ & $99^{\circ} 54^{\prime} 42.7^{\prime \prime}$ & 14 & 1888 & $29-5$ al 29-9 \\
\hline 42 & $20^{\circ} 36^{\prime} 25^{\prime \prime}$ & $99^{\circ} 54^{\prime} 43.1^{\prime \prime}$ & 12 & 1927 & $02-6$ al $13-10$ \\
\hline 43 & $20^{\circ} 35^{\prime} 9.1^{\prime \prime}$ & $99^{\circ} 54^{\prime} 35.3^{\prime \prime}$ & 12 & 1919 & $02-6$ al $13-10$ \\
\hline 44 & $20^{\circ} 30^{\prime} 13.3^{\prime \prime}$ & $99^{\circ} 54^{\prime} 40.2^{\prime \prime}$ & 14 & 1902 & $29-5$ al 29-9 \\
\hline 46 & $20^{\circ} 29^{\prime} 41.3^{\prime \prime}$ & $99^{\circ} 55^{\prime} 54.8^{\prime \prime}$ & 14 & 1887 & $29-5$ al 29-9 \\
\hline 47 & $20^{\circ} 27^{\prime} 47.6^{\prime \prime}$ & $99^{\circ} 56^{\prime} 56.2^{\prime \prime}$ & 15 & 1900 & $29-5$ al 29-9 \\
\hline
\end{tabular}

Fuente: trabajo en campo y con datos climáticos de CONAGUA

Nota. En el cuadro no aparecen los puntos 2, 4,3.5.12 y 30, porque se localizan fuera del Mpio.

El cuadro 4, muestra de manera detallada las características de la forma de las tierras: pendiente, textura, profundidad, $\mathrm{pH}$, reacción del suelo al $\mathrm{HCl}$ para estimar la presencia de carbonato. Para medir la materia orgánica se usa el agua oxigenada. Además, se estima la cantidad de piedras y rocas en porciento. Asimismo, se calcula la cubierta vegetal, las unidades de suelo. Con todo lo anterior y los aspectos climáticos se calcula erosión hídrica. Así como las clases de tierra por cada sitio de muestreo. En los 17 agroambientes se obtienen datos en 47 sitios. Los datos de los agroambientes 4 y 10 se obtienen con información de gabinete, de la Síntesis Geográfica de Querétaro, INEGI (1995). En el agroambiente 13 no hay datos, porque es la parte urbana del municipio 


\begin{tabular}{|c|c|c|c|c|c|c|c|c|}
\hline : & 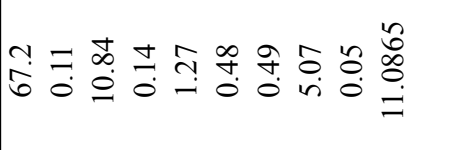 & 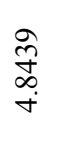 & $\begin{array}{l}\stackrel{\circ}{n} \\
\stackrel{n}{\Xi} \\
=\end{array}$ & $\begin{array}{l}n \\
\infty \\
n\end{array}$ & 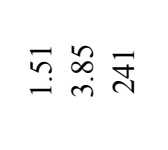 & 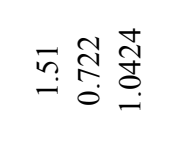 & $\stackrel{m}{\stackrel{0}{0}}$ & 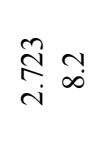 \\
\hline 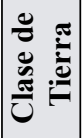 & 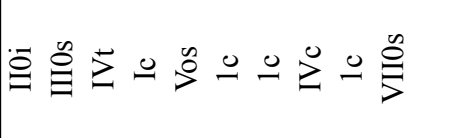 & $\stackrel{\infty}{>}$ & $\stackrel{e}{\ominus}$ & 兑 & $0 \stackrel{\infty}{0} \stackrel{\infty}{\rho} \stackrel{0}{\theta}$ & 000 & $\stackrel{8}{\varrho}$ & $\stackrel{0}{\varrho}$ \\
\hline 宽 & 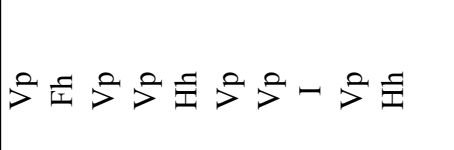 & 国 & 寻 & 靑 & 띠 피 & 山 $>^{2}>^{2}$ & $\simeq$ & 平 $\simeq$ \\
\hline 胥 & 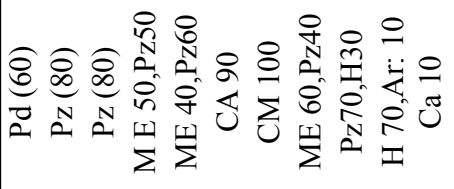 & 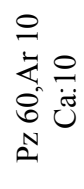 & 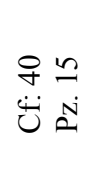 & & 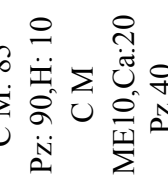 & 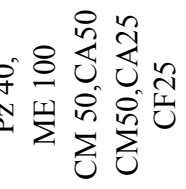 & 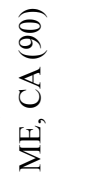 & 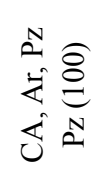 \\
\hline$\underbrace{\varrho}_{\simeq}$ & $\approx 000000 ? 0 \mathrm{~N}$ & $\cong$ & $\stackrel{0}{z}$ & $\stackrel{\circ}{z}$ & 000 & 000 & 0 & $0 ?$ \\
\hline$\overbrace{}^{\infty}$ & 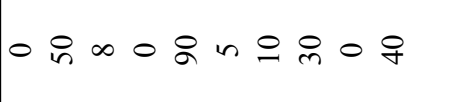 & 尺 & n & $\sim$ & N & n no & \& & 유으 \\
\hline (ே) & 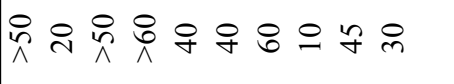 & \& & $\stackrel{n}{\wedge}$ & \& & $\stackrel{n}{\wedge} \stackrel{+}{\sim}$ & 요소 & 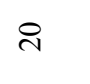 & 유으 \\
\hline है & 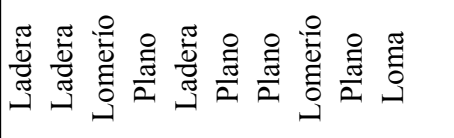 & 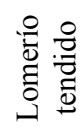 & 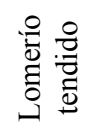 & 을 & 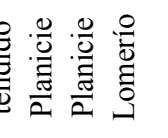 & 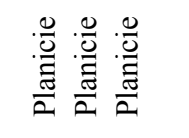 & 율 율 & 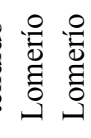 \\
\hline$\underbrace{\varrho}_{\Xi}$ & $0 O \therefore \circ \sigma m \sim \pm n$ & $\infty$ & $\sim$ & $\nabla$ & 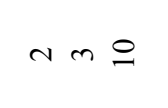 & $N-7$ & n & $\infty$ ণ \\
\hline$\stackrel{\pi}{4}$ & $\infty \because 0 \pm \backsim b \pm \simeq \pm a$ & $a$ & $a$ & $a$ & $\Xi \Xi \pm$ & $\pm-n$ & n & $-\simeq$ \\
\hline$\stackrel{\ominus}{\Xi}$ & $-n b+\infty a \circ=m \pm$ & 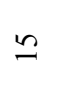 & 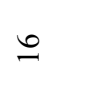 & 듬 & $\stackrel{2}{2}$ & $\vec{\sim} \approx$ & $\stackrel{\sim}{\sim}$ & $\approx 2$ \\
\hline
\end{tabular}


Genaro Aguilar-Sánchez

Differentiation of agricultural lands in the municipality of Tequisquiapan, Querétaro

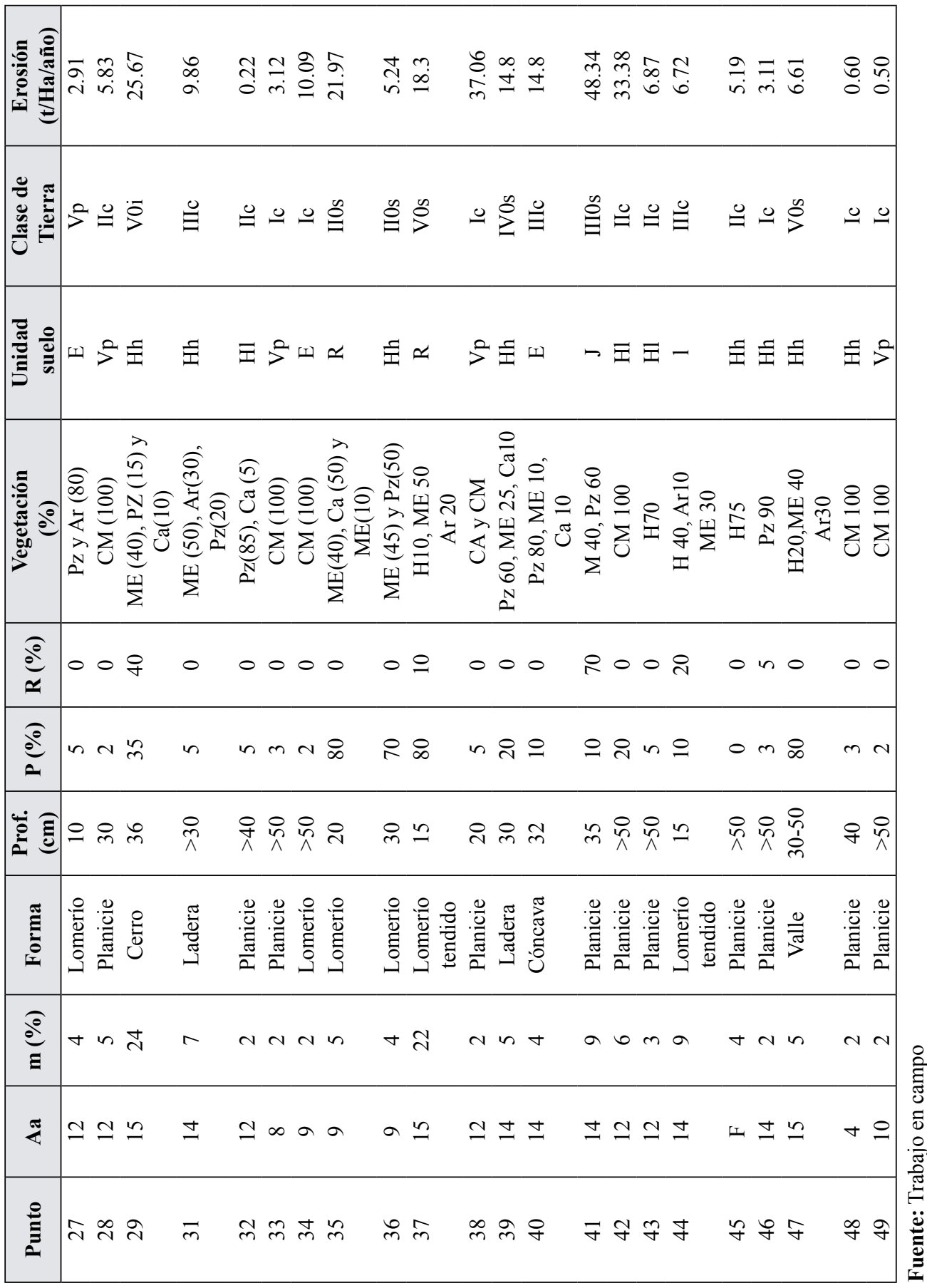


En el cuadro no aparecen los puntos 2,4,3.5.12 y 30, porque se localizan fuera del Mpio.

Aa: agroambiente, $\mathrm{m}$ : pendiente, $\mathrm{P}$ : pedregosidad, R: rocosidad, M.O: Materia Orgánica.

$\mathrm{HCl}$ : ácido clorhídrico al $10 \%$, se usa para detectar la presencia de carbonatos en el suelo,

NR: No reacciono, RM: Reacción media, RA: Reacción alta

$\mathrm{pH}$ : Se mide con tiras de plástico $\mathrm{pH}$, con rangos de 0.0 a 14.0

Cuadro 5. Significado de las literales usadas en el cuadro 4.

Diferenciación de clases de tierras

\begin{tabular}{|c|c|c|}
\hline Vegetación & Suelo & Limitante en tierra \\
\hline Pz: pastizal & I: litosol & c: clima \\
ME: matorral espinoso & R: regosol & p: profundidad \\
Ca: cactáceas & E: rendzina & $\begin{array}{c}\text { 0i: obstrucción interna dada por la } \\
\text { rococidad, }\end{array}$ \\
H: herbáceas & J: fluvisol & $\begin{array}{c}\text { Os: obstrucción superficial } \\
\text { (pedregosidad) }\end{array}$ \\
Ar: Arboles & Vp: vertisol pelico & \\
Pd: plantación de durazno & Hh: feozem haplico \\
CM: cultivo de maíz & Hl: feozem luvico & \\
CA: cultivo de alfalfa & & \\
Cc: cultivo de cebolla & & \\
Cf: cultivo de frijol & & \\
Cav: Cultivo de avena & \\
Cf: Cultivo de flor & & \\
\hline
\end{tabular}

La diferenciación de tierras agrícolas se realizó como se indica en la metodología, siguiendo la propuesta de la (FAO, 1997).

En el municipio de Tequisquiapan se identificaron siete clases de tierras, tomando en cuanta toda la información de gabinete y con los puntos de muestreo. La clasificación de tierras, se puede observar en el cuadro 4, que van de la clase I a la Clase VII, con limitantes manifiestas de alta cantidad de rocas y piedras, topografía, clima y profundidad del suelo, las que se describen a continuación. 
De acuerdo a los datos obtenidos, la clase I, es la más adecuada para realizar agricultura, la mayoría de los puntos catalogados en esta clase cuentan con superficies de riego, pendientes ligeras, así como mayor profundidad en los suelos. Los puntos determinados en la clase II, coinciden en tener una pendiente de 4 a $7 \%$, así como una disminución considerable de profundidad en los suelos, y el nivel de pedregosidad comienza a ser evidente en la superficie.

En la clase III encontramos áreas con mayor dificultad de acceso por la pendiente, la cual abarca del 8 al $12 \%$, y una mayor población forestal. Otra característica de esta clase es la alta susceptibilidad a la erosión. Una de las restricciones que se identificó en un punto fue el alto porcentaje de pedregosidad por lo cual se obtiene la subclase III s.

La clase IV en Tequisquiapan presenta pendientes muy pronunciadas entre 13 y $20 \%$, con un desarrollo de cultivos limitado. La pedregosidad que se observa está entre 11 a $20 \%$. Se observó susceptibilidad a la erosión. Presenta suelos poco profundos, en este caso la concentración de materia orgánica es alta. Para la clase IVs la única restricción que presenta es la alta rococidad. En estas clases de tierra se observó actividad pecuaria. La clase $\mathrm{V}$ se definió por la presencia de pedregosidad y rococidad mayor a $20 \%$, aunque se encontraron pendientes no tan ligeras, el factor de obstrucción superior e interior son grandes limitantes para el desarrollo de cultivos. Se observó la presencia de pastizales y actividades pecuarias.

Clase VI, son tierras con pendientes adecuadas para soportar una vegetación permanente. Con suelos que deben permanecer bajo bosque, bien sea natural o plantado. No son adecuados para ningún tipo de cultivo a causa de procesos erosivos severos y muy poca profundidad efectiva. Las pendientes suelen ser mayores del $25 \%$. La explotación ganadera debe hacerse de forma extensiva muy controlada, bajo sistemas silvopastoriles y en ocasiones es necesario dejar los terrenos desocupados por largos periodos de tiempo para su recuperación. En estas tierras son necesarias prácticas de recuperación de suelos como terrazas, terrazas de inundación, filtros y drenajes en espina de pescado, y que exista vegetación permanente.

Clase VII, son tierras con pendientes mayores del $25 \%$ y restricciones muy fuertes por pedregosidad, rococidad, baja fertilidad, suelos muy superficiales, litosol y regosol, y erosión de media a alta. Son áreas de protección que deben permanecer cubiertas por vegetación densa de matorral 
espinoso, y bosque de encino. Su principal uso es para la protección de suelos, aguas, flora y fauna. Solo son aptos para mantener coberturas arbóreas permanentes.

\section{Análisis y Conclusiones}

En la clasificación de tierras agrícolas, interacciones de los aspectos de relieve, clima, geología, suelo, y el uso actual. La combinación de las características de los cinco aspectos nos muestra una gran diversidad de tierras, que por afinidad los podemos agrupar en clases, a escala detallada 1:50,000 o a nivel 1:10,000, o incluso si queremos un detalle particular lo podemos reducir al tamaño de una parcela de 1 ha. De manera general, las clases de tierras más afines se pueden agrupar por agroambientes, a escala $1: 250,000$.

El clima es relevante para el ciclo agrícola de los cultivos, por lo que para los agricultores que siembran con lluvia del temporal, es vital conocer los cambios del clima en el tiempo y en los diversos espacios, (Granados R., 2004; Bautista, 2016), para poder sembrar con menos incertidumbre, por lo mismo los 4 PC obtenidos en los 17 agroambientes son aceptables a escala general.

La erosión hídrica que se obtiene en los 49 sitios de muestreo y los 17 agroambientes, es una estimación que ayuda a diagnosticar el estado actual de la perdida de suelo, sin embargo, vale comentar que la aplicación de la Ecuación Universal de Pérdida de Suelo, EUPS, tiene la limitante de la aplicación de la ecuación general para estimar la erosividad de la lluvia, (Colegio de Postgraduados, 1991), y que la EUPS se realizó en condiciones particulares como lo indica (Wischmeir, 1978). Sin embargo, la EUPS, si nos ayuda a conocer la erosión en unidades cartográficas generales (Aguilar, G., y Estrada, J 1982)

La geología contribuye a la formación de suelo, al igual que la vegetación, en función de lo anterior la geología de rocas ígneas extrusivas y basalto, contribuye a la formación y predominancia de suelos I, R, Hh, y $\mathrm{Vp}$, Y sólo existe suelo E, en tres agroambientes. La pendiente de más del $10 \%$ y la presencia de la vegetación en los lomeríos y ladera de los cerros, junto con las lluvias torrenciales, se combinan para que exista erosión media. En contraparte en áreas planas del menos de $5 \%$ de inclinación la erosión es poca. 
Así tenemos que todos los elementos del medio natural y el uso actual de las tierras contribuye a que la clasificación de tierras, fluctué de clase I, cuya limitante es el clima semiseco, al igual que las clases de II y III, donde la profundidad del suelo y la presencia de un porcentaje pequeño de piedras son poco limitantes, para la producción agrícola de maíz, frijol, o para huertas de nopal, durazno, uva, etc. En las clases IV y V, además de las piedras, poca profundidad, clima semiseco, tiene más relevancia la inclinación del terreno de más del $15 \%$. Tierras que se pueden usar para el pastoreo de ganado extensivo, o semiestabulado, controlando la rotación de potreros. En las clases VI y VII cobra importancia la presencia media y alta de rocas, $>50 \%$ de pedregosidad; así como una inclinación mayor del $25 \%$, tierras que se recomienda se usen para la preservación de la vegetación, o un uso controlado de cultivos de la región como huertas de nopal, durazno o de maguey.

El trabajo es aceptable a escala 1:250,000, sin embargo, para tener mayor certeza es necesario hacer una investigación a escala 1:20,000, pero tendríamos la limitante de la falta de datos climáticos más precisos y, realizar el muestreo de suelo más intensivo.

\section{Referencias}

Aguilar S, G. y Estrada B.J. (1982). Perdidas de suelo en México. Chapingo, 79-84.

Aguilar Sánchez, G. (1995). Diferenciación agroclimática de la región Queréndaro-Morelia, Michoacán, México. México, DF: Insituto de Geografía-UNAM.

Bautista, F. (2016). Zonificaciòn agroclimatica de la región de infiernillo. Biodiversidad del estado de Michoacán. México, DF.: CONABIO.

Becerra Moreno, A. (1999). Escorrentía, erosión, y conservación de suelos. Texcoco, Estado de México: UACh.

Colegio de Postgraduados (1991). Manual de predicción de perdidas de suelo por erosión. México, DF: SARH.

CONAGUA (14 de Agosto de 2016). www.conagua.gob.mx. Obtenido de CONAGUA.

Edo de Qurètaro. (18 de Septiembre de 2016). Querétaro está entre nosotros. Recuperado de http://www.queretaro.gob.mx/municipios.aspx. Obtenido de queretaro.gob. 
FAO. (1997). Boletín de suelos de la FAO: Zonificación agroecologica. Roma Italia: ONU.

Fernández Reynoso, D. S. (2015). Estimación de la erosión del suelo. Texcoco, Edo. Mèxico: UACh.

Granados R., R. T. (2004). Aptitud agroclimatica en la Mesa Central de Guanajuato, México. México, DF: Intituto de Geografía-UNAM.

INAFED (2010). Enciclopedia de municipios. Tequisquiapan. Querétaro, Qro.: Edo.Qro.

INEGI (1995). Sintesis geogràfica del estado de Querètaro. México, DF: Inegi.

INEGI (2005). Uso potencial del suelo. Aguascalientes, Ags: INEGI.

INEGI (2015). Anuario estadístico y geográfico del estado de Querétaro. México: INEGI.

Lafuente, C. (2008). Metodologías de la investigación en las ciencias sociales: fases,fuentes y selección de temas. México: Escuela de Administración de Negocios.

Matías, L. F. O. \&. G. F. Centro Nacional de Prevención de Desastres (2001). Heladas. Ciudad de México.

Ortiz, C., \& Cuanalo, H. (1984). Metodología del Levantamiento Fisiográfico, un sistema de clasificación de tierra. Texcoco, México: COLPOS.

Pájaro, D. (1988). Estimación del Periodo de Crecimiento por disponibilidad y libre de herladas para la República Mexicana. Estado de México: Colegio de Postgraduados: Centro de Edafología.

Rojas, S. (2012). Métodos para la investigación social. México D.F: Plaza y Valdés.

Romero Peñaloza, J. (1992). El condicionamiento ambiental y el anàlisis regional de la agricultura; una experiencia. Texcoco, estado de México: Direcciòn de Centros Regionales-UACh.

Romero, P.J. (2003). Agricultura y recursos naturales en la cuenca del lago de Cuitzeo, Michoacán. Cuaderno de Centro Regionales No. 25. Chapingo, México.: DCRU-UACH,.

Sánchez, F.(24 de Noviembre de 2016 Noviembre ). Cálculo de la Precipitación areal mediante Sistemas de Información Geográfica. [En línea] Recuperado de: http://api.eoi.es/api_v1_dev.php/fedora/asset/ eoi:45221/componente45219.pdf . Obtenido de /api.eoi.es. 
SEDEA-Qro. (15 de 7 de 2018). Recuperado de www. sedea.queretaro. gob.mx/regiones. Obtenido de sedea queretaro.

SIAP., 2003-2015 (15 de agosto de 2016). SIAP 2003-2015. Recuperado de www.Servicio de Información Agroalimentaria.. Obtenido de www. infosiap.gob.mx/agricola siao gb/ientidad/index.jsp.inade

UNAL (2016). Marco conceptual de la erosión. Bogota, Colombia: Uni. Nal. de Colombia.

Universidad Autonòma de Querétaro. (1995). Enciclopedia Temática del Estado de Querétaro, Tomo 1 Geografia de Querétaro. Mèxico, DF. Talleres de Artículos Gráficos de México, S.A.

USDA.. (1962). Land-capability Classification, Soil Conservation Service U.S. Washington:: Departament of Agriculture.

Wischmeir, W. A. (1978). Predicting rainfall erosionlosses a guide to conservation planning. Whasington D.C.: USDA. 
